\title{
Latest Developments in Multiple Ion Species Plasma FIB Technology
}

\author{
Brandon Van Leer ${ }^{2}$ and Mikhail Dutka
}

1. Thermo Fisher Scientific, Hillsboro, OR, USA.

2. Thermo Fisher Scientific, Materials \& Structural Analysis, Eindhoven, the Netherlands.

Compared to $\mathrm{Ga}^{+}$liquid metal ion source (LMIS) DualBeam FIB-SEMs, $\mathrm{Xe}^{+}$inductively couple plasma (ICP) DualBeams are relatively new [1]. Until recently, only $\mathrm{Ga}^{+}$and $\mathrm{Xe}^{+}$source technologies have been commercially available. LMIS $\mathrm{Ga}^{+}$FIB technology offers an ion beam that provides high resolution capability for nanoprototyping and offers enough current for S/TEM sample preparation of most samples in less than an hour as well as FIB serial sectioning tomography (SST) of volumes up to $40 \mathrm{X} 40 \mathrm{X} 40 \mu \mathrm{m}^{3} / \mathrm{hr}$ (silicon using $30 \mathrm{kV}-65 \mathrm{nA}$ ) with interslice distances as small as $3 \mathrm{~nm}$ [2,3]. On the other hand, $\mathrm{Xe}^{+} \mathrm{PFIB}^{3}$ technology has a much larger beam current capability allowing researchers to investigate volumes up to $130 \mathrm{X} 130 \mathrm{X} 130 \mu \mathrm{m}^{3} / \mathrm{hr}$ (silicon using $30 \mathrm{kV}-2.5 \mu \mathrm{A}$ ). Additionally, xenon ions offer the user the ability to prepare gallium-free S/TEM samples or cross-sections [4,5]. However, for a number of applications, neither gallium nor xenon may provide the highest quality results because of their intrinsic properties.

In this paper, we present the latest generation of plasma FIB technology supporting multiple ion species as a primary ion beam on Thermo Fisher's newest DualBeam, the Helios Hydra PFIB. Next to xenon, the Helios Hydra provides researchers with 3 additional ion species beyond xenon - argon, oxygen and nitrogen. A single ion source can deliver all 4-ion species independently with a patented, automated, fast and easy switching capability. Thermo Fisher expects that access to multiple ion species "on-the-fly" will significantly expand the FIB's application space in addition to providing advantages to various use cases. For example, consider S/TEM sample preparation with $\mathrm{Xe}^{+}$ions and a final low energy polish of $\mathrm{Ar}^{+}$ions to improve contrast in HR-STEM.

The Helios Hydra PFIB is designed on an established high-performance platform, the Helios NanoLab, offering state-of-the-art SEM performance with sub-nm imaging at $1 \mathrm{keV}$ and up. The multiple ion source technology allows for gas switching between species in less than 10 minutes without any degradation in performance for $\mathrm{Xe}^{+}$PFIB. The system is fully compatible with the complete line up of automation from Thermo Fisher: AutoTEM 4, Auto Slice\&View 4, AutoScript 4, Maps and iFAST.

\section{References:}

[1] P Tesch et al., Proceedings from the $34^{\text {th }}$ International Symposium for Testing and Failure Analysis (2008) p 6.

[2] CA Volkert and AM Minor, MRS Bulletin 32 (2007), p.389.

[3] J Mayer et al., MRS Bulletin 32 (2007), p. 400.

[4] LA Giannuzzi and N Smith, Microscopy and Microanalysis 17 (2011), p. 646.

[5] B Van Leer and R Passey, Microscopy and Microanalysis 23 (2017), p. 272. 


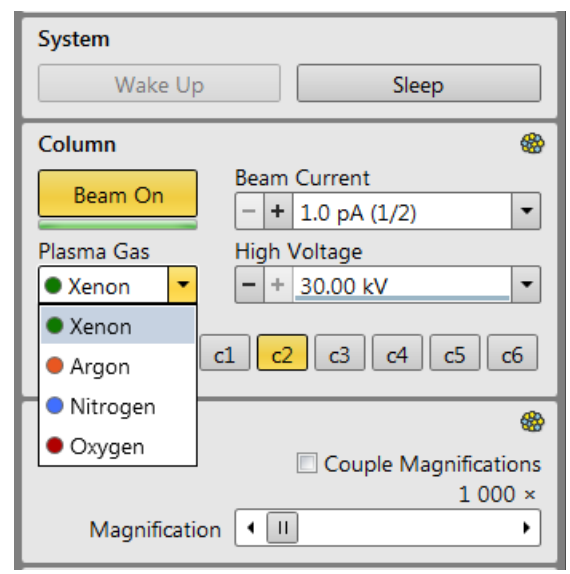

Figure 1. Graphical User Interface of the Helios Hydra PFIB showing user selectable plasma gas.

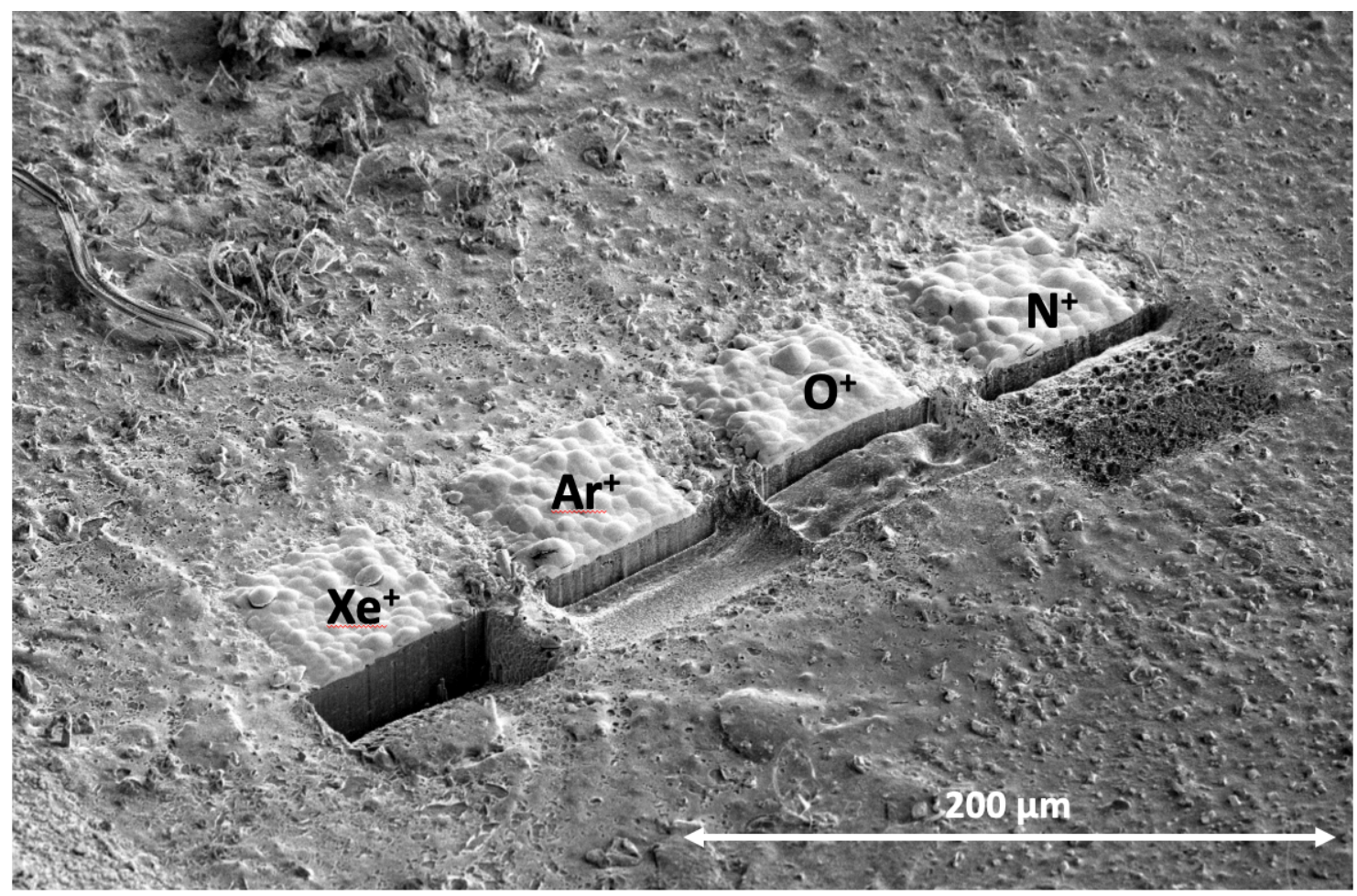

Figure 2. Example of cross-sections conductive rubber prepared using differ ion species at $30 \mathrm{kV}$. 